\title{
Effect of Endoplasmic Reticulum Stress on Inflammation and Adiponectin Regulation in Human Adipocytes
}

\author{
Ashis K. Mondal, Ph.D., ${ }^{1}$ Swapan K. Das, Ph.D.,' Vijayalakshmi Varma, Ph.D., ${ }^{2}$ Greg T. Nolen, M.S., ${ }^{2}$ \\ Robert E. McGehee, Ph.D., ${ }^{3}$ Steven C. Elbein, M.D., Jeanne Y. Wei, M.D., Ph.D., ${ }^{4}$ and Gouri Ranganathan, Ph.D. ${ }^{4}$
}

\begin{abstract}
The endoplasmic reticulum (ER) of adipocytes plays a major role in the assembly and secretion of adipokines. The levels of serum adiponectin, secreted by adipocytes, are decreased in insulin resistance, diabetes, and obesity. The role of ER stress in downregulating adiponectin levels has been demonstrated in mouse models of obesity. Studies examining human adipose tissue have indicated that there is an increase in the ER stress transcript HSPA5 with increased body mass index (BMI). However, it is not established whether ER stress results in changes in adiponectin levels or multimerization in human adipocytes. We examined whether the induction of ER stress using tunicamycin, thapsigargin, or palmitate alters the messenger RNA (mRNA) and protein expression of adiponectin and the mRNA expression of chaperones ERP44 and ERO1 in adult-derived human adipocyte stem (ADHAS) cells. ER stress was measured using key indicators of ER stress-HSPA5, ERN1, CHOP, and GADD34, as well as changes in eIF2 $\alpha$ phosphorylation. Because ER stress is suggested to be the proximal cause of inflammation in adipocytes, we further examined the change in inflammatory status by quantitating the change in Iк $\beta-\alpha$ protein following the induction of ER stress. Our studies indicate that: (1) ER stress markers were increased to a higher degree using tunicamycin or thapsigargin compared to palmitate; (2) ER stress significantly decreased adiponectin mRNA in response to tunicamycin and thapsigargin, but palmitate did not decrease adiponectin mRNA levels. In all three instances, the induction of ER stress was accompanied by a decrease in adiponectin protein as well as adiponectin multimerization. All three inducers of ER stress increased tumor necrosis factor- $\alpha(\mathrm{TNF}-\alpha)$ mRNA and decreased Iк $\beta-\alpha$ protein in adipocytes. The data suggest that ER stress modifies adiponectin secretion and induces inflammation in ADHAS cells.
\end{abstract}

\section{Introduction}

A LTHOUGH THE PATHOPYSIOLOGICAL mechanisms that link obesity with type 2 diabetes mellitus (T2DM) are not known, obesity has been identified as the most prevalent risk factor. Recent literature suggests that obesity results in a state of chronic inflammation of the expanding adipose tissue, which is characterized by altered adipokine secretion and ultimately manifests as metabolic syndrome. Endoplasmic reticulum (ER) stress has been proposed as the immediate cause of chronic inflammation and reduced insulin action at the molecular, cellular, and systemic levels. ${ }^{1}$ The exact mechanism(s) by which chronic inflammation of the adipose tissue might result in decreased insulin sensitivity and metabolic syndrome are not completely understood. In- flammation of the adipose tissue might be the cause or the result of changes in insulin sensitivity. The ER is the continuation of the nuclear membrane and the site for the synthesis and folding of both membrane-associated and secreted proteins. Abnormal conditions such as nutrient deprivation, elevated glucose, or lipids can disrupt ER homeostasis and lead to accumulation of unfolded or misfolded proteins in the ER lumen. This is especially observed for cells that make highlevels of secretory proteins and require an evolved mechanism to properly fold, process, and release the proteins. ${ }^{2}$

Adiponectin, an adipokine produced exclusively and at high levels by adipocytes, is an important mediator of both metabolic and antiinflammatory effects that correlate with insulin sensitivity. ${ }^{3}$ Numerous studies have demonstrated that adiponectin levels are low in patients with T2DM,

\footnotetext{
${ }^{1}$ Wake Forest School of Medicine, Winston-Salem, North Carolina.

${ }^{2}$ Division of Personalized Nutrition and Medicine, National Center for Toxicological Research, Jefferson, Arkansas.

${ }^{3}$ Department of Pediatrics and ${ }^{4}$ The Central Arkansas Veterans Healthcare System and Department of Geriatrics, University of Arkansas for Medical Sciences, Little Rock, Arkansas.
} 
metabolic syndrome, insulin resistance, and cardiovascular disease. ${ }^{4}$ Adiponectin is assembled and secreted by adipocytes in several different multimeric isoforms, including low molecular weight (LMW) (trimer), medium molecular weight (MMW) (hexamer), and high molecular weight (HMW) (18-mer and higher). The expression of adiponectin in adipocytes is regulated both at the transcriptional and posttranscriptional steps by a variety of regulatory factors. ${ }^{5}$ In humans, the ratio of HMW adiponectin to LMW adiponectin is correlated with insulin sensitivity. ${ }^{6,7}$

It has been suggested that ER stress plays a causative role in the association of obesity and insulin resistance with T2DM. ${ }^{8}$ We hypothesized that the induction of ER stress in adipocytes could alter the expression and secretion of adiponectin by human adipocytes. Therefore, we examined the induction of ER stress by measuring key indicators of ER stress response (ERSR) following treatment with tunicamycin, thapsigargin, or palmitate and quantitated the resulting changes in adiponectin expression and secretion by adipocytes. The induction of ER stress downregulated adiponectin processing and secretion and increased tumor necrosis factor$\alpha(\mathrm{TNF}-\alpha)$ in human adipocytes. In addition ER stress decreased Iк $\beta-\alpha$, a marker of inflammation in human adipocytes.

\section{Methods}

\section{Cell culture}

Adult-derived human adipocyte stem (ADHAS) cells were isolated using discarded adipose tissue from normal women undergoing liposuction according to procedures described previously. 9,10 Subjects provided informed consent under protocols that were approved by the local institutional review board. ADHAS adipocytes were obtained by differentiation of preadipocytes as described previously. ${ }^{11}$ Briefly, ADHAS cells were grown and maintained in Dulbecco modified Eagle medium (DMEM)/F12 medium (Gibco) containing $10 \%$ fetal calf serum (FCS), penicillin (100 IU/ $\mathrm{mL})$, and streptomycin $(100 \mu \mathrm{g} / \mathrm{mL})$. For experimental purposes, cells were plated at $0.4 \times 10^{5}$ cells per well in six-well plates and maintained in growth medium until 1 day postconfluence, followed by differentiation using medium containing $250 \mu \mathrm{M}$ 3-isobutyl-1-methylxanthine (Sigma), $1 \mu \mathrm{M}$ rosiglitazone , $100 \mathrm{nM}$ insulin, $1 \mu \mathrm{M}$ dexamethasone (Sigma), $33 \mu \mathrm{M}$ biotin (Sigma), $17 \mu \mathrm{M}$ pantothenic acid (Sigma), and $3 \%$ serum. Cells were incubated in the differentiation medium for 3 days. Cell media were then changed to an adipogenic medium consisting of $100 \mathrm{nM}$ insulin, $1 \mu \mathrm{M}$ dexamethasone (Sigma), $33 \mu \mathrm{M}$ biotin (Sigma), $17 \mu \mathrm{M}$ pantothenic acid (Sigma), and 3\% serum for 12-13 days when morphologically differentiated adipocytes were obtained. Following hormonal stimulation, greater than $60 \%$ of these cells underwent complete differentiation into mature adipocytes as assessed by Oil Red O lipid staining.

\section{Reagents}

Rosiglitazone (Takada Pharmaceuticals, Deerfeild, IL), isobutryl-methylxanthene, pantothenic acid, biotin, and dexamethasone (Sigma, St. Louis, MO) were added to the differentiation medium just before use. Stock solutions for tunicamycin $(1 \mathrm{mg} / \mathrm{mL})$ (Sigma, St. Louis, MO), palmitate $(100 \mathrm{mM})$, and thapsigargin $(1 \mathrm{mg} / \mathrm{mL})$ were dissolved in ethanol or dimethylsulfoxide (DMSO). Palmitate was conju- gated to fatty acid free bovine serum albumin (BSA; dissolved in DMEM cell culture medium) at a 2.5:1 molar ratio by incubating the mixture at $45^{\circ} \mathrm{C}$ for $20 \mathrm{~min}$ and then filter sterilizing before adding the mixture to adipocyte cultures. Differentiated adipocytes were treated with tunicamycin $(1 \mu \mathrm{g} / \mathrm{mL})$, thapsigargin $(25 \mathrm{nM})$, vehicle, palmitate-BSA conjugate $(500 \mu \mathrm{M})$, or the same concentration of BSA in serum-free culture medium for $18 \mathrm{~h}$ as described. ${ }^{12}$ Following the described treatment, cells were washed in serum-free culture medium at $4^{\circ} \mathrm{C}$ and lysed with RNA lysis buffer for RNA or with RIPA buffer containing protease inhibitors for protein extraction.

\section{RNA isolation and gene expression}

Total RNA from cultured adipocytes was isolated using RNAqueous kit (Ambion, Austin, TX) according to the manufacturer's instructions. RNA was quantitated spectrophotometrically and quality was examined using agarose gels. Total RNA (500 ng) was reverse transcribed using random hexamer primers and TaqMan Reverse Transcription Reagents (Applied Biosystems, Foster City, CA). Reversetranscribed RNA [complementary DNA (cDNA)] was amplified with $1 \times$ SYBR Green PCR Master Mix (Applied Biosystems, Foster City, CA) using $0.3 \mu \mathrm{M}$ gene-specific primers and an ABI 7900 RT PCR system. Primer sequences were designed to span an intron and are described below. Samples were normalized to $18 \mathrm{~S}$ ribosomal RNA. Standard curves were generated using pooled cDNA from the samples being compared. Triplicate measurements were performed for analysis. XBP1 splicing was examined by PCR amplification of the reverse-transcribed RNA (cDNA) for total and spliced $\mathrm{XBP} 1$, followed by gel electrophoresis and quantification of the bands by densitometric analysis.

\section{Primer description}

Primers were designed as follows: 18S-F, 5'-ATCAACTTT CGATGGTAGTCG-3', 18S-R, 5'-TCCTTGGATGTGGTAG CCG-3'; HSPA5-F, 5'-GGTATTGAAACTGTGGGAGGTG-3', HSPA5 R, 5'-TTGTCTTTTGTCAGGGGTCTTT-3'; ERN1-F, 5'-ACACCATCACCATGTACGACACCA-3', ERN1-R: 5'-AT TCACTGTCCACAGTCACCACCA-3'; CHOP-F, 5'AGGGA GAACCAGGAAACGGAAACA-3', CHOP-R, 5'-TCCTGCTT GAGCCGTTCATTCTCT-3'; GADD34-F, 5'-CTAGGCTGCC CCTCCGAC-3', GADD34-R, 5"-CTCGGAGAAGCGCACCT TTCTG-3'; XBP1-F* , 5'-GCTGAAGAGGAGGCGGAAG-3', XBP1R* , 5'-GTCCAGAATGCCCAACAGG-3'; Adiponectin-F, 5'-ATGCCCAAAGAGGAGAGAGGAA 3', Adiponectin-R, 5'-TGGTCAGAAACAGGCACACAAC 3'; ERp44-F, 5'-AGG TGCCGCTGCCTGGAGAA-3' ' ERp44-R, 5'-CGGCTGGGAC TGGGCTAGGT-3', 5' -ATGCCCAAAGAGGAGAGAGGAA3'; TNF- $\alpha-F$, 5'-GGATCATTGCCCTGTGAGGA-3', TNF- $\alpha-\mathrm{R}$, 5'-TTTGAGCCAGAAGAGGTTGAG-3'.

Primer sequences marked with an asterisk $\left({ }^{*}\right)$ were used to test XBP1-unspliced (US) and spliced (S) on 2.5\% agarose gel. All other primers were used for SYBR Green-based quantitative PCR.

\section{Immunoblot analysis}

Following treatment for $20 \mathrm{~h}$, medium and cells were collected for analysis. ADHAS cells were washed in phosphate-buffered saline (PBS) followed by lysis using $1 \times$ RIPA 
lysis buffer (Santa Cruz Biotechnology Inc., Santa Cruz, CA) [1× Tris-buffered saline (TBS), 1\% NP-40, 0.5\% sodium deoxycholate, supplemented with protease and phosphatase inhibitors]. Cell lysates were homogenized, and total protein was determined using Bradford protein assay reagent (Sigma, St Louis, MO). To detect changes in JNK1 and eIF2 $\alpha$ phosphorylation, equal quantities of each sample $(40-50 \mu \mathrm{g}$ protein) was denatured by boiling with sample buffer containing $1 \% \quad \beta$-mercaptoethanol and $2 \%$ sodium dodecyl sulfate (SDS), followed by separation through an $8 \%$ SDSpolyacrylamide gel.

To detect changes in adiponectin multimerization, equal quantities of medium or cell lysates were separated using nondenaturing $4 \%-15 \%$ gradient gels. When examining the effect of palmitate treatment, due to the presence of a large amount of BSA in the medium, a smaller fraction of medium was separated using nondenaturing gels as compared to the tunicamycin or thapsigargin treatments. To detect changes in total adiponectin, cell lysates (5-10 $\mu$ g protein) were denatured with sample buffer containing 1\% $\beta$-mercaptoethanol and $2 \%$ SDS and separated through a 10\% SDS-polyacrylamide gel.

After separation by SDS-polyacrylamide gel electrophoresis (PAGE), the proteins were transferred onto a Trans-Blot nitrocellulose membrane (Bio-Rad Inc., CA), which was hybridized with a specific primary antibody followed by secondary antibody. The reaction products were visualized with chemiluminescence reagents (Amersham, Piscataway, NJ). The blots were scanned, and densitometric analysis was performed using Quantity One Image Analysis software (v4.6.3, Bio-Rad). To ensure equal loading of protein, the blots were normalized to $\beta$-actin or glyceraldehyde 3-phosphate dehydrogenase (GAPDH), by probing each blot subsequently with $\beta$-actin or GAPDH antibody. Primary antibodies for western blot experiments included phosphoeIF2 $\alpha$ (BioSource Division of Invitrogen, Carlsbad, CA), total eIF2 $\alpha$, GAPDH, $\beta$-actin (Santa Cruz Biotechnology, Inc. Santa Cruz, CA), adiponectin (R\&D Systems, Minneapolis, $\mathrm{MN}), \mathrm{I} \kappa \beta-\alpha$ (Cell Signaling Technology, Inc. Danvers, MA), and horseradish peroxidase (HRP)-conjugated secondary antibodies (Amersham Biosciences Pittsburgh, PA).

\section{Statistical analysis}

Data are presented as means \pm standard deviation (SD). Protein expression levels were compared across different gels after taking a ratio of specific protein/ $\beta$-actin and using the two-tailed Student's $t$ test. Statistical significance was set at $P \leq 0.05$.

\section{Results \\ Effect of different ER stressors on ER stress response (ERSR) transcripts in human adipocytes}

The induction of ER stress pathways was examined by measuring changes in HSPA5, ERN1, CHOP, and GADD34 transcripts as well as XBP1 mRNA splicing. Figure 1A shows the expression of HSPA5, ERN1, CHOP, and GADD34 normalized to $18 \mathrm{~S}$ RNA. The ER stress genes that were examined were comparable between the DMSO-treated control and the BSA vehicle-treated control. ER resident chaperone protein HSPA5, which is a key mediator of ER stress response, was increased 20-fold and 30-fold by tunicamycin and thapsigargin treatments, respectively, as compared to
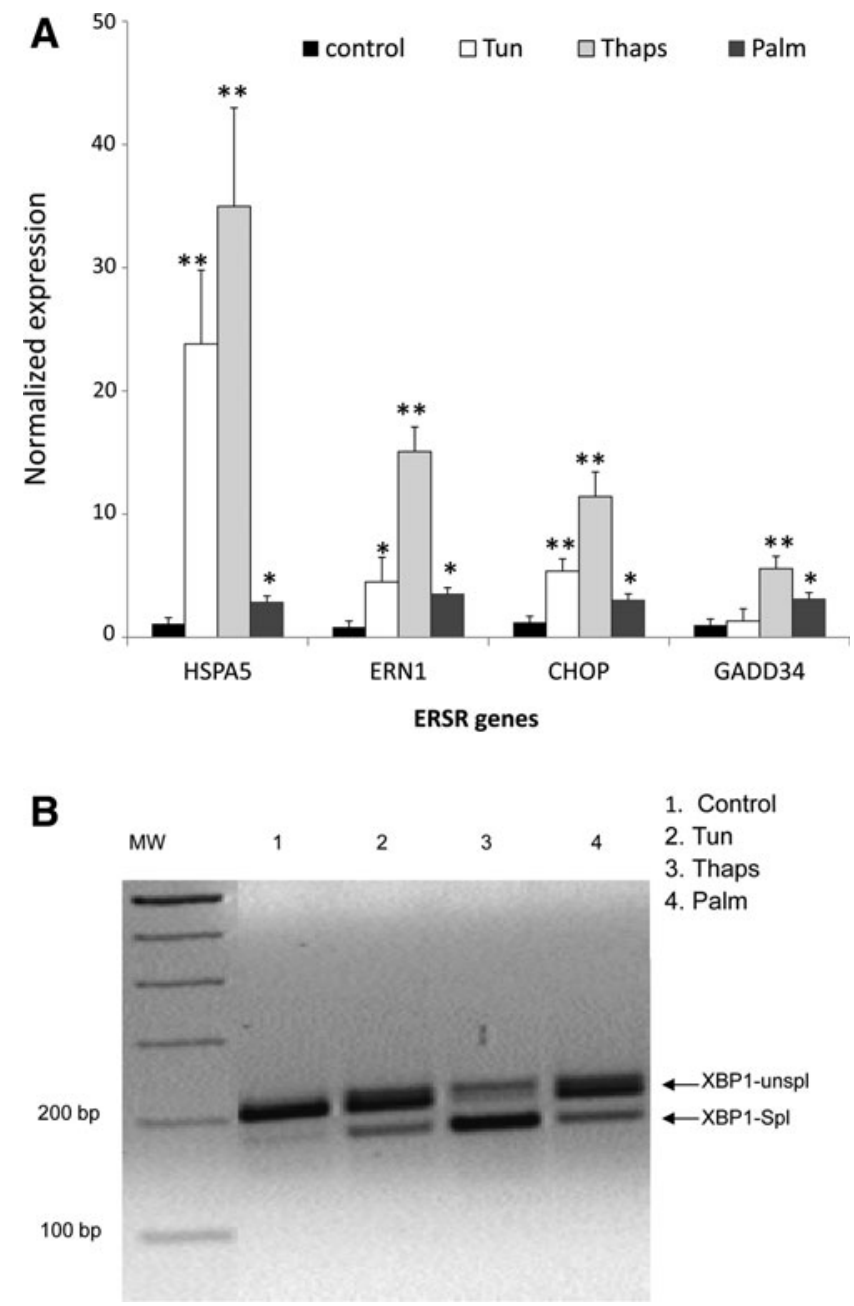

FIG. 1. Effect of tunicamycin (Tun), thapsigargin (Thaps), or palmitate (Palm) on endoplasmic reticulum (ER) stress markers in human adipocytes. (A) Expression of ER stress pathway genes in adipocytes following $18 \mathrm{~h}$ of treatment with tunicamycin $(1 \mu \mathrm{g} / \mathrm{mL})$, thapsigargin $(25 \mathrm{nM})$, palmitate $(500 \mu \mathrm{M})$, or control [dimethylsulfoxide (DMSO) or bovine serum albumin (BSA)]. Expression values shown are obtained from real-time measurements of each transcript normalized to 18S RNA. Data are presented as means \pm standard deviation (SD) of $18 \mathrm{~S}$ normalized expression from of two independent experiments with three biological replicates $\left({ }^{* *} P<0.003,{ }^{*} P<0.05\right)$. (B) Representative agarose gel showing spliced and unspliced XBP1 transcripts from the control (lane 1), tunicamycin- (lane 2), thapsigargin- (lane 3), and palmitate- (lane 4) treated samples described in A. ERSR, Endoplasmic reticulum stress response; MW, molecular weight marker; unspl, unspliced; Spl, spliced.

the vehicle control, whereas palmitate treatment increased HSPA5 expression by 3- to 4-fold over the vehicle control. ERN1, which encodes the ER stress response protein IRE1 and the transcription factor CCAAT/enhancer-binding protein-homologous (CHOP), were upregulated 3- to 4-fold by tunicamycin and palmitate and more than 10 -fold by thapsigargin treatment. The growth arrest and DNA damage indicator transcript GADD34 was upregulated 3- to 4-fold by thapsigargin or palmitate treatments, but was not changed by tunicamycin treatment. Splicing of XBP1 is a marker of acute ER stress; XBP1 splicing was quantitated by measuring 


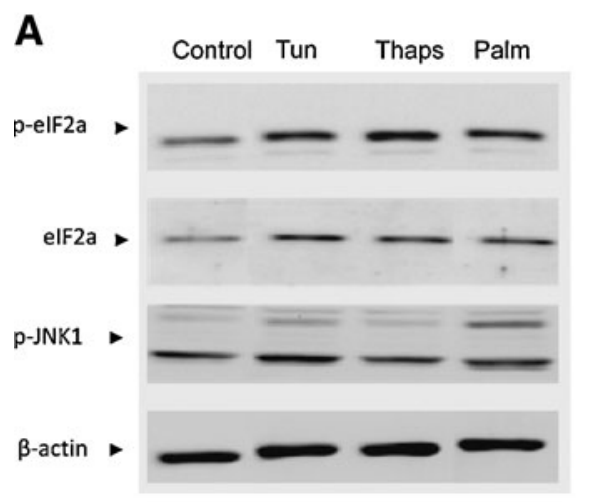

B
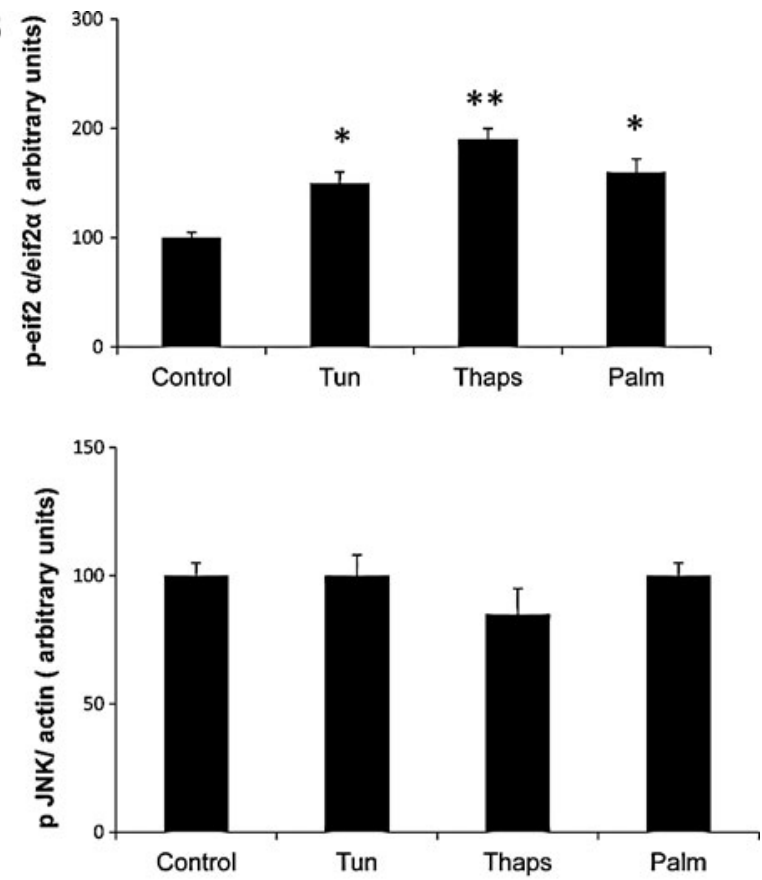

FIG. 2. Effect of different endoplasmic reticulum (ER) stressors on eIF2 $\alpha$ and JNK1 phosphorylation in human adipocytes. (A) Western blot of cell lysates from control, thapsigargin (Thaps)-, and tunicamycin (Tun)-treated adipocytes showing phosphorylated and unphosphorylated eucarayotic initiation factor eIF $2 \alpha$, pJNK1, and $\beta$-actin from a representative experiment performed as in Fig. 1. (B) Quantitation of p-eIF $\alpha 2 /$ eIF2] $\alpha$ and p-JNK1/ $\beta$-actin from experiments described in Fig. 1. Data are plotted as mean and \pm standard deviation (SD) of the control from experiments described in Fig. $1\left({ }^{*} P<0.05\right.$, ** $P<0.03)$. Palm, Palmitate.

the percent of the unspliced to spliced XBP1 product. All three agents increased XBP1 splicing by more than $30 \%$ (data not shown). Figure $1 \mathrm{~B}$ is a representative gel showing the change in XBP1 splicing following treatment with tunicamycin, thapsigargin, or palmitate. Thapsigargin was the strongest inducer of ER stress transcripts and XBP1 splicing.

\section{Effect of different ER stressors on JNK1 and elF2 $\alpha$ phosphorylation in human adipocytes}

Obesity-induced ER stress is known to cause the phosphorylation and activation of PERK (encoded by eIF2 $\alpha \mathrm{K} 3$ ), which in turn phosphorylates eIF $2 \alpha$, resulting in a halt to protein translation. ER stress is also known to trigger phosphorylation and activation of JNK1, resulting in inhibition of insulin receptor signaling. ${ }^{1}$ Das et al. have demonstrated that palmitate-induced ER stress triggers the phosphorylation of
eIF2 $\alpha$ and JNK1 in HepG2 cells. ${ }^{12}$ As shown in Fig. 2A, all three agents increased the ratio of p-eIF- $2 \alpha /$ eIF $2 \alpha$, tunicamycin and palmitate increased p-eIF- $2 \alpha$ by $40 \pm 5 \%$, and thapsigargin increased p-eIF- $2 \alpha$ by $70 \pm 5 \%$, but there was no change in JNK1 activation in adipocytes treated with ER stress agents for $18 \mathrm{~h}$. Data are plotted as arbitrary units relative to the mean of the control (vehicle treatment) (Fig. 2B).

\section{Effect of ER stress on adiponectin multimerization}

Adiponectin is secreted and exists in circulation as trimers (LMW), hexamers (MMW), and HMW forms. The HMW multimers of adiponectin are more bioactive, and the ratio of $\mathrm{HMW} /$ total adiponectin rather than the measure of total adiponectin is a better indicator of insulin sensitivity. ${ }^{6}$ Figure 3A shows the changes in adiponectin in the medium and cell

FIG. 3. Effect of endoplasmic reticulum (ER) stress on adiponectin multimerization. (A) Western blots of medium (lanes M) $(25 \mu \mathrm{L}$ of $1 \mathrm{~mL})$ and cell lysates (lanes C) $(10 \mu \mathrm{g}$ protein) were separated through a $5 \%-15 \%$ nonreducing gradient minisodium dodecyl sulfate polyacrylamide gel showing multimeric forms of adiponectin from control [dimethylsulfoxide (DMSO)], thapsigargin (Thaps)-, and tunicamycin (Tun)-treated adipocytes described in Fig. 1. (B) Quantitation of highmolecular-weight (HMW), medium-molecular-weight (MMW), and low-molecular-weight (LMW) adiponectin in medium (M) fraction shown in A. Data are plotted as arbitrary units relative to the mean \pm standard deviation (SD) of the control (Con) $\left({ }^{*}\right.$ < 0.001$)$. (C) Quantitation of HMW, MMW, and LMW adiponectin in cell lysates (C) shown in A. Data are plotted as arbitrary units relative to the mean \pm SD of the control $\left({ }^{*} P<0.05\right)$. (D) Western blot of cell lysates from control, thapsigargin-, and tunicamycin-treated adipocytes from experiments described in Fig. 1. Samples were boiled with sample buffer containing $50 \mathrm{mM} \beta$-mercaptoethanol followed by separation through a 10\% SDS-polyacrylamide gel to detect the change in total adiponectin and glyceraldehyde 3-phosphate dehydrogenase (GAPDH). (E) Quantitation of total adiponectin/GAPDH in cell lysate is shown in $\mathbf{D}$. Data are plotted as arbitrary units relative to the mean of the control $\pm \mathrm{SD}\left({ }^{*} P<0.005\right)$. 
A
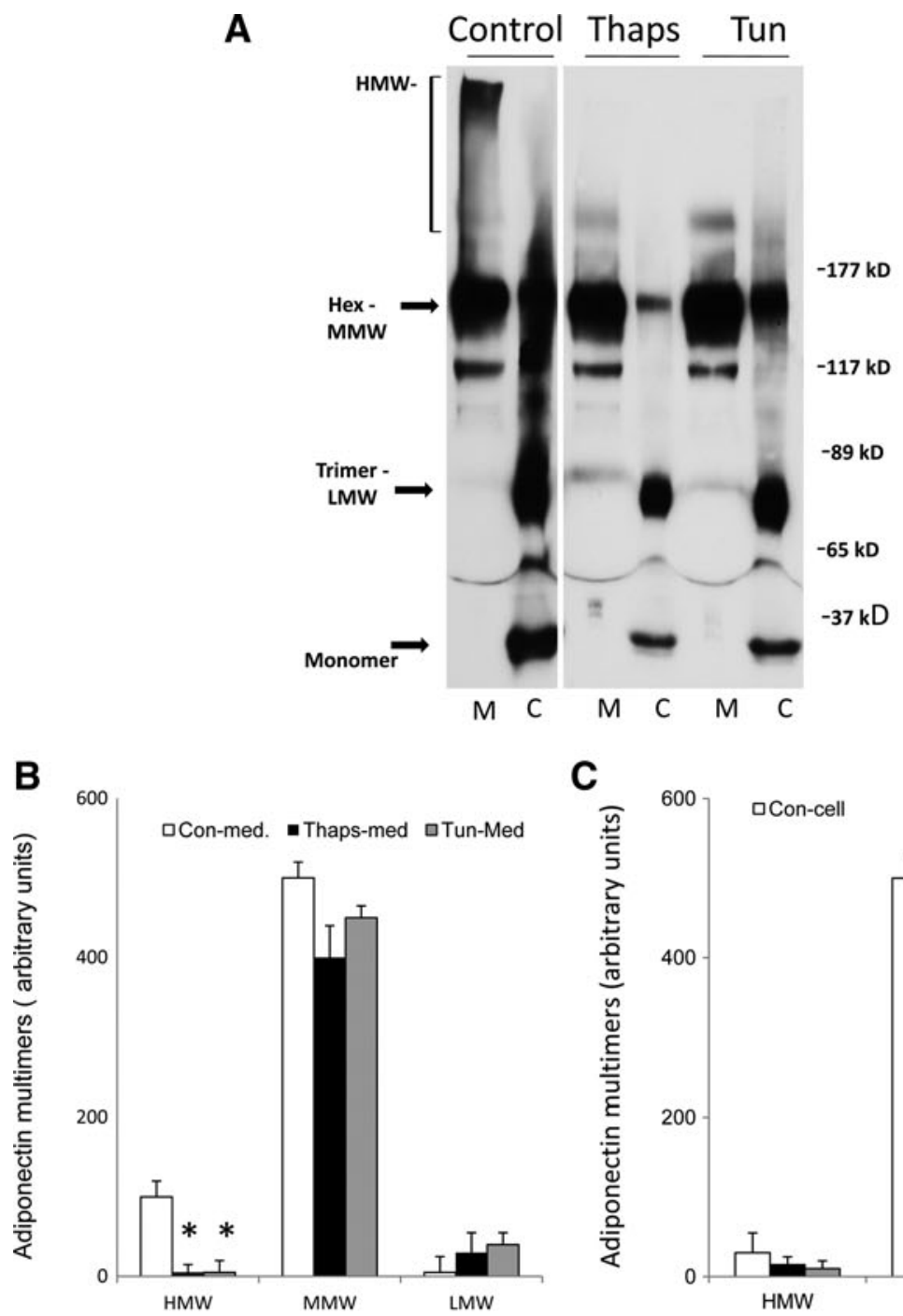

C

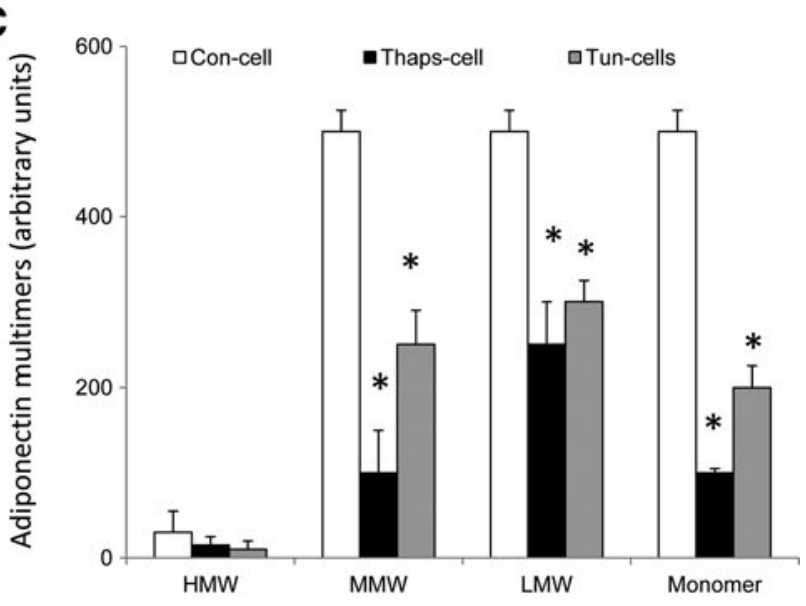

D
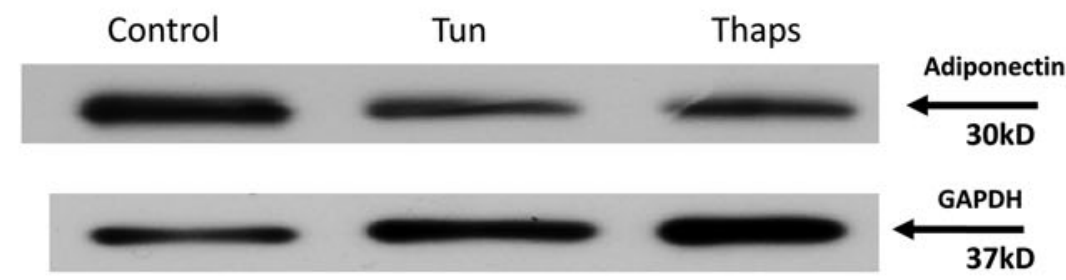

E

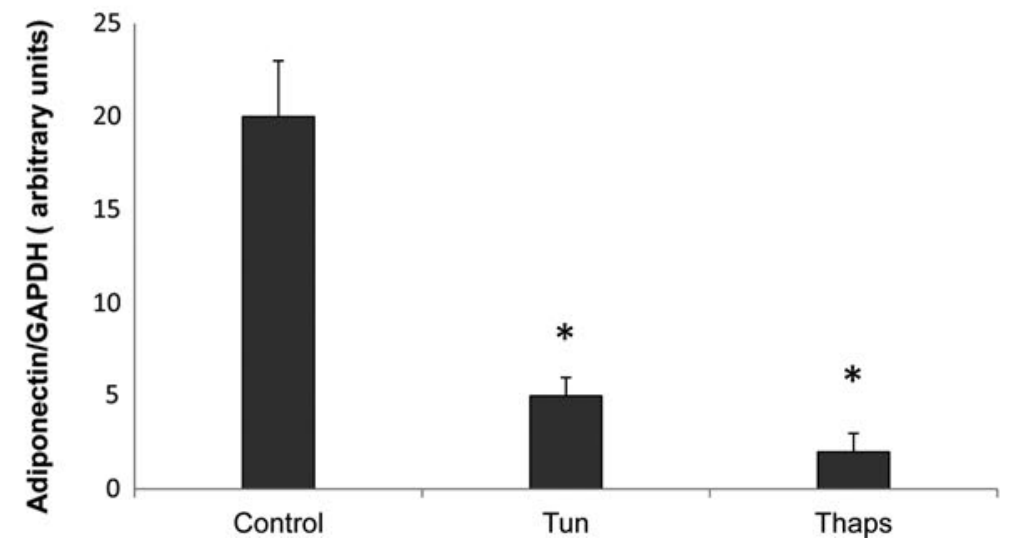


lysate following treatment of ADHAS cells-derived adipocytes with tunicamycin or thapsigargin. Quantitation of adiponectin in the medium indicated the following changes: HMW adiponectin was decreased by $90 \% \pm 5 \%$ following treatment with thapsigargin. MMW adiponectin was the most abundantly expressed form in the medium and this decreased by $20 \% \pm 10 \%$ following treatment with thapsigargin or tunicamycin, which, however, was not significant. The LMW adiponectin was the least expressed, and treatment with tunicamycin or thapsigargin increased LMW to a small extent; however, this increase was not significant (Fig. 3B). The HMW isoform was detected to a small extent in control cell lysate, and this was further decreased by tunicamycin and thapsigargin treatments. The MMW, LMW, and monomers were all detected prominently in control cell lysates. Tunicamycin inhibited MMW adiponectin by $50 \% \pm 5 \%$ and thapsigargin inhibited MMW adiponectin by $80 \% \pm 5 \%$ (Fig. 3C). LMW adiponectin was decreased by $50 \% \pm 15 \%$ by both tunicamycin and thapsigargin, and adiponectin monomer was decreased by $50 \% \pm 10 \%$ by both tunicamycin and thapsigargin treatments (Fig. 3C).

The cell lysates were also examined for change in total adiponectin (Fig. 3D). The ratio of total adiponectin/GAPDH protein was inhibited by $75 \% \pm 5 \%$ following tunicamycin treatment and $85 \% \pm 5 \%$ following thapsigargin treatment (Fig. 3E). ER stress induction by thapsigargin or tunicamycin decreased total adiponectin protein and HMW adiponectin in the medium.

Induction of ER stress using palmitate was compared to cells treated with BSA conjugate (Fig. 4A,B). Quantitation of adiponectin in the medium indicated that palmitate treatment decreased the formation of HMW adiponectin by $80 \% \pm 5 \%$, MMW adiponectin showed a tendency to increase, whereas LMW adiponectin was barely detectable in medium and did not change (Fig. 4A,C). In the cell lysates, palmitate treatment decreased HMW adiponectin by $80 \% \pm 5 \%$ and MMW adiponectin by $50 \% \pm 10 \%$, but LMW was not significantly altered (Fig. 4B,D). To examine changes in total adiponectin, cell lysates were examined. Palmitate treatment inhibited total adiponectin by $60 \% \pm 5 \%$ (Fig. $4 \mathrm{E}, \mathrm{F}$ ). Thus, ER stress induction using palmitate decreased HMW adiponectin secretion as well as total adiponectin protein.

\section{Changes in adiponectin, ER chaperones, and TNF- $\alpha$ following induction of ER stress}

During protein synthesis, the synthesized protein is translocated to the ER almost simultaneously and the proteins are bound by molecular chaperones. The chaperone Erp44 regulates the secretion of adiponectin by thiolmediated retention, and together with Ero1 $\alpha$ and Ero1 $\beta$ promotes disulfide bond formation, resulting in secretion of various polymeric forms of adiponectin and retention of unpolymerized molecules. ${ }^{13}$ We examined the change in adiponectin $\mathrm{mRNA}$ and adiponectin-related chaperone ERP44 and ERO1 $\alpha$ as well as TNF- $\alpha$, an inflammatory cytokine, following induction of ER stress using tunicamycin, thapsigargin, or palmitate treatments. Adiponectin mRNA was inhibited by $60 \% \pm 5 \%$ after tunicamycin or thapsigargin (Fig. 5A), but the induction of ER stress using saturated fatty acid palmitate did not alter adiponectin mRNA levels (Fig. 5A). ERp44 levels were increased following tunicamycin or thapsigargin by 3 - to 5 -fold, but palmitate treatment did not increase ERp44 (Fig. 5A); however ERO1 levels were increased by more than $25 \% \pm 5 \%$ following induction of ER stress with either tunicamycin, thapsigargin, or palmitate. TNF- $\alpha$ mRNA was dramatically increased by tunicamycin, thapsigargin, and palmitate treatments. The increase in TNF- $\alpha$ mRNA as shown in Fig. 5A was more than 5-fold in all three instances.

\section{Changes in inflammatory signaling following induction of ER stress}

We examined whether ER stress induction resulted in increased inflammation by examining the levels of Ік $\beta-\alpha$ in cells treated with tunicamycin, thapsigargin, or palmitate. Both tunicamycin and thapsigargin decreased Ік $\beta-\alpha$ levels in ADHAS cell-derived adipocytes by $80 \% \pm 10 \%$ (Fig. 5B). Palmitate treatment also decreased Iк $\beta-\alpha$ by about $75 \% \pm 5 \%$ as compared to the BSA-treated control (Fig. 5C). Thus, all three inducers of ER stress also increased inflammation in human ADHAS-derived adipocytes.

\section{Conclusions}

A number of mechanisms, including inflammation and ER stress, are thought to play a causal role in the development of obesity-related metabolic disorders. ${ }^{4,14}$ Studies using animal models have demonstrated that obesity-linked metabolic disorders downregulate adipocytokine secretion from adipose tissues. ${ }^{15,16}$ Adipose tissues of obese mice are hypoxic and express an increase in ER stress markers, CHOP, and GRP78, along with decreased expression of adiponectin mRNA. ${ }^{17}$ Similar studies performed using obese $d b / d b$ mice also demonstrated that relieving ER stress using the chemical chaperone TUDCA reduced CHOP protein, which was induced by obesity and increased circulating levels of adiponectin. ${ }^{18}$ Obesity-induced ER stress has been linked to inflammatory responses and increased peripheral insulin resistance. ${ }^{19}$ Mitochondrial dysfunction and ER stress have been proposed as independent determinants of insulin resistance as well as obesity. ${ }^{1,20}$ It has been shown that mitochondrial dysfunction can cause ER stress in adipose tissues and result in decreased adiponectin transcription in obese $d b /$ $d b$ mice. ${ }^{21}$ Other reports also indicate a role for inflammatory cytokines in the induction of ER stress and the downregulation of insulin-sensitizing hormone adiponectin in transgenic mouse models of obesity. ${ }^{22}$

Studies by Das et al. on subcutaneous adipose tissues of human subjects indicate that, although pioglitazone treatment improved insulin sensitivity significantly and increased adiponectin levels, it did not alter subcutaneous adipose tissue expression of ER stress genes HSPA5, ATF6, CHOP, $E R N 1$, or $X B P 1 .{ }^{12}$ However, other studies by the same group also indicated that the expression of genes involved in inflammation and ER stress significantly correlated with the percent fat mass. ${ }^{23}$ The mechanism by which ER stress may affect the expression of adipocytokines is not clearly established. To examine whether the induction of ER stress in human adipocytes triggers alterations in adiponectin mRNA and protein, we treated ADAHS cell-derived adipocytes with different ER stress-inducing agents such as tunicamycin (glycosylation inhibitor), thapsigargin $\left(\mathrm{Ca}^{2}\right.$-ATPase inhibitor), or palmitate (saturated fatty acid). ER stress induction was measured by quantitation of changes in HSPA5, ERN1, 

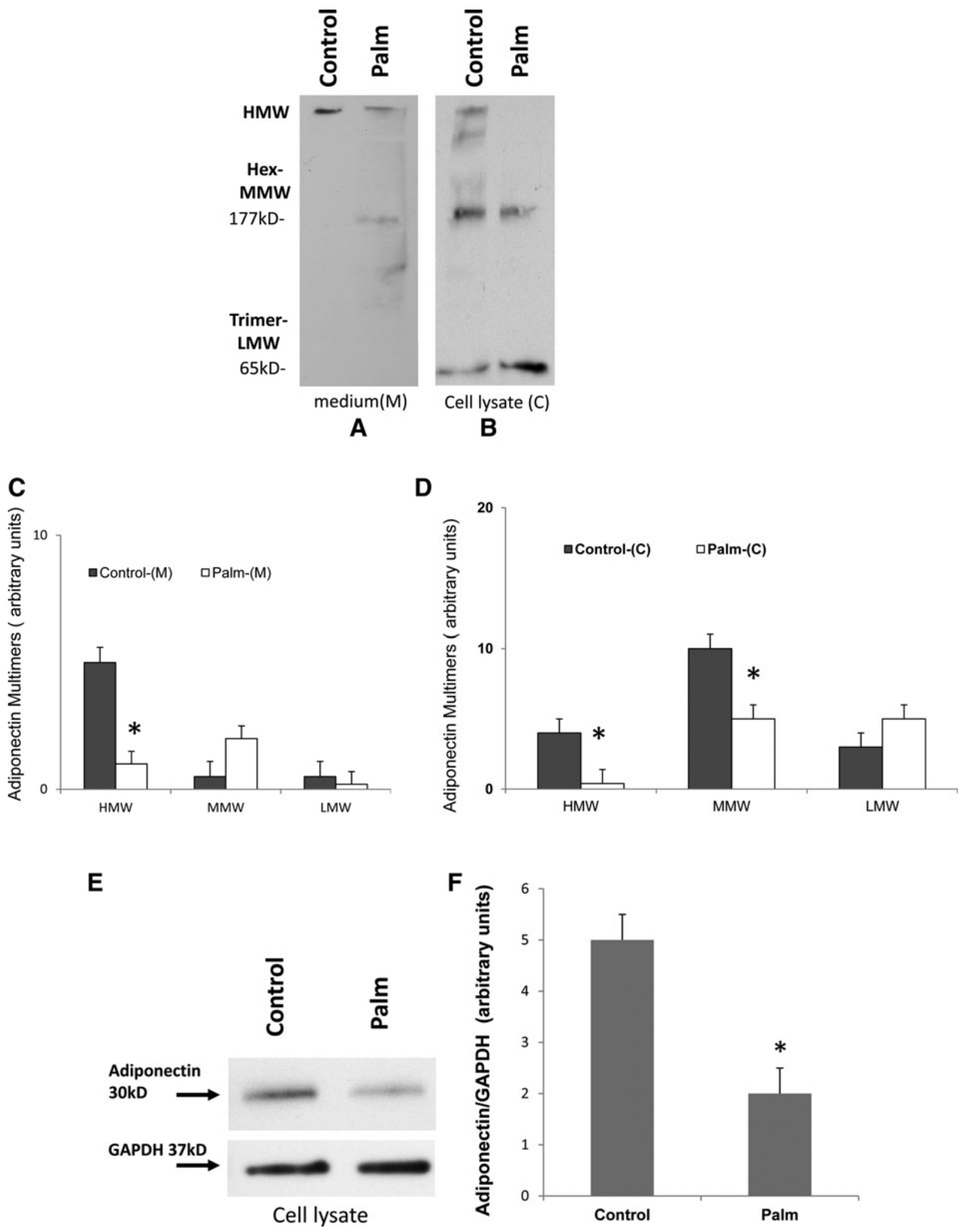

FIG. 4. Effect of endoplasmic reticulum (ER) stress following palmitate (Palm) treatment on adiponectin multimerization. Western blot of medium ( $5 \mu \mathrm{L}$ of $1 \mathrm{~mL})$ (A) and cell lysates $(2 \mu \mathrm{g}$ protein) (B) separated using a $5 \%-15 \%$ gradient mini-sodium dodecyl sulfate (SDS)-polyacrylamide gel showing multimeric forms of adiponectin from control (bovine serum albumin, BSA) or palmitate-treated adipocytes described in Fig. 1. (C and D) Quantitation of high-molecular-weight (HMW), mediummolecular-weight (MMW), and low-molecular-weight (LMW) adiponectin shown in A and B. Data are plotted as arbitrary units relative to the mean \pm standard deviation (SD) of the control $\left({ }^{*} P<0.05\right)$. (E) Western blots of cell lysates from control and palmitate-treated adipocytes. Samples were boiled in sample buffer containing $50 \mathrm{mM} \beta$-mercaptoethanol followed by separation through a 10\% SDS-polyacrylamide gel, to detect the change in total adiponectin and glyceraldehyde 3-phosphate dehydrogenase (GAPDH). (F) Quantitation of total adiponectin/GAPDH in cell lysate shown in E. Data are plotted as arbitrary units relative to the mean \pm SD of the control $\left({ }^{*} P<0.005\right)$. 

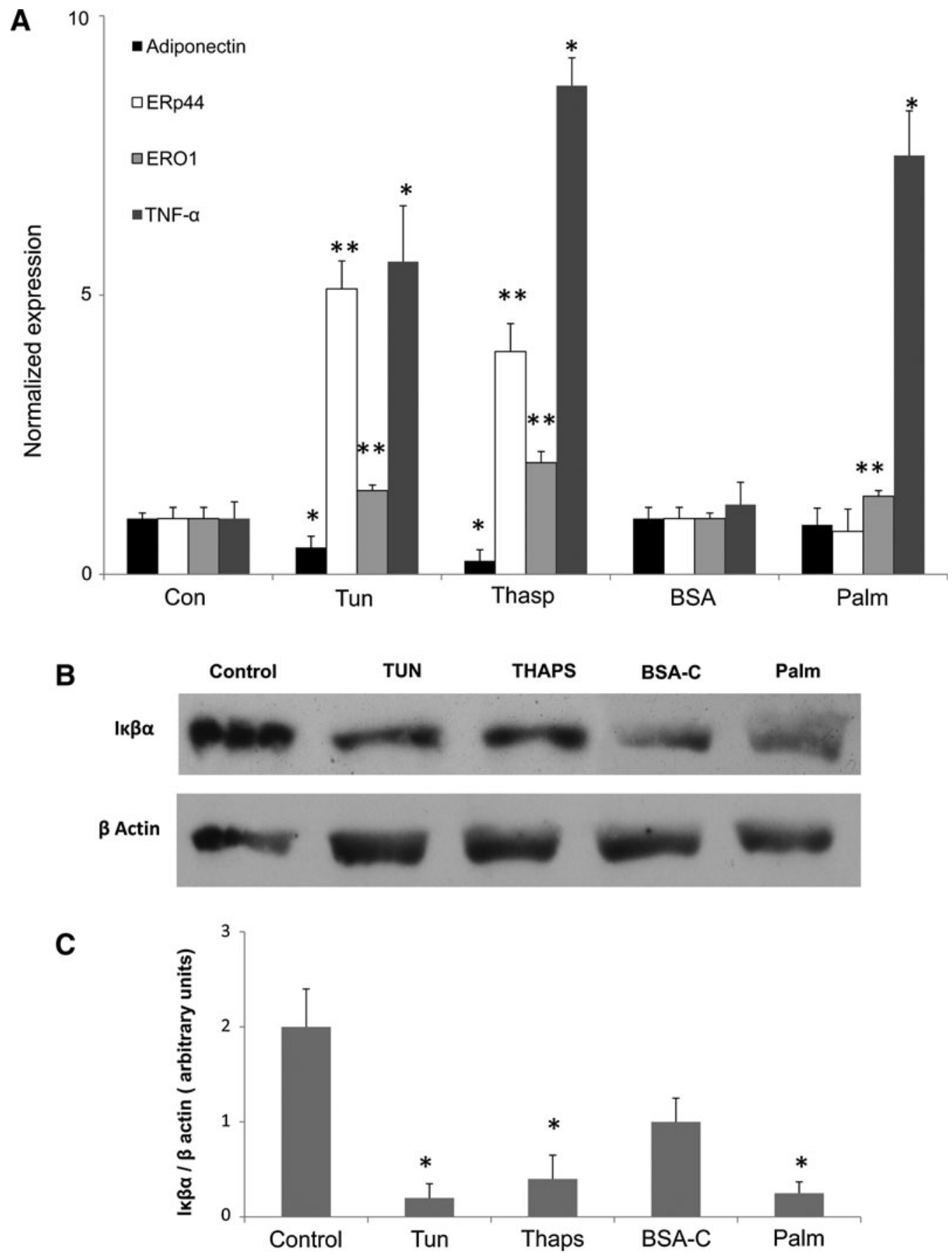

FIG. 5. Changes in adiponectin, endoplasmic reticulum (ER) chaperones, tumor necrosis factor- $\alpha$ (TNF- $\alpha$ ) messenger RNA (mRNA), and inflammation indicator Iк $\beta-\alpha$ following induction of ER stress. (A) Expression of adiponectin, ERP44, ERO1, and TNF- $\alpha$ mRNA in adipocytes following $18 \mathrm{~h}$ of treatment with tunicamycin (tun), thapsigargin (thasp), palmitate (Palm), or control ([dimethylsulfoxide (DMSO) or bovine serum albumin (BSA)]. Expression values of each transcript are obtained by real-time measurements normalized to $18 \mathrm{~S}$ RNA measurements. Data are presented as means \pm standard deviation (SD) of $18 \mathrm{~S}$ normalized expression from of three independent experiments with two biological replicates $\left({ }^{*} P<0.05,{ }^{* *} P<0.01\right)$. (B) Western blot of cell lysates from control (DMSO)-, tunicamycin-, thapsigargin-, control (BSA)-, or palmitate-treated adultderived human adipocyte stem (ADHAS) cell-derived adipocytes to detect change in I $\kappa \beta-\alpha$. The same blot was probed for $\beta$-actin as a loading control. (C) Quantitation of change in I $\kappa \beta-\alpha / \beta$-actin shown in B. Data are plotted as arbitrary units relative to the mean $\pm \mathrm{SD}$ of the control $\left({ }^{*} P<0.05\right)$.

CHOP, GADD34, and XBP1 mRNA as well as measuring changes in phosphorylation of target proteins eIF2 $\alpha$ and JNK1. We observed that all the ER stress-inducing agents augmented the expression of marker genes of ER stress HSPA5, ATF6, CHOP, and ERN1 and spliced XBP1 mRNA and increased eIF2 $\alpha$ phosphorylation. However, we did not find an increase in JNK1 activation; it is likely that JNK1 activation occurred at an earlier interval after induction of ER stress. Studies by Sharma et al. comparing adipose expression of eIF- $2 \alpha$ and JNK1 in subjects with low and high body 
mass index (BMI) have shown an increase in eIF2 $\alpha$ phosphorylation along with an increase in ER stress transcripts with increased BMI, but no significant change in JNK1 activation was observed. ${ }^{24}$

It is known that the ER proteins PERK, ATF6, and IRE-1 are maintained in an inactive state by binding with ERresident chaperones GRP78 and GRP94. ER stress can cause the accumulation of misfolded proteins in the ER lumen; when the chaperones are occupied by misfolded proteins, this results in the release and activation of the ER stress sensors PERK, ATF6, and IRE-1 and subsequent triggering of downstream signaling pathways of ER stress. ${ }^{25}$ Cells respond by triggering eIF2 $\alpha$ phosphorylation, which in turn halts general protein translation so that the cell can expend reserves on cell survival processes. An augmentation of ER stress genes as well as eIF $2 \alpha$ phosphorylation was observed when ADHAS cell-derived adipocytes were subjected to ER stress in this study. Simultaneous to the ER stress response, there was also a significant decrease in adiponectin secretion in the medium and in cell lysates, demonstrating that the induction of ER stress resulted in a decrease in the HMW fractions as well as total adiponectin protein in human adipocytes. Total adiponectin as well as HMW adiponectin are thought to play a role in insulin sensitivity as well as metabolic syndrome. ${ }^{26,27}$

Although general protein synthesis is halted by p-eIF $2 \alpha$, translation of GADD34 mRNA apparently proceeds unhindered. GADD34 is a stress-induced protein that promotes dephosphorylation of eIF $2 \alpha$ and restores normal cell function. ${ }^{28}$ We found that both tunicamycin and thapsigargin are inducers of ER stress gene expression in human adipocytes, and the induction of HSPA5, ERN1, GADD34, and CHOP mRNA is similar to the levels of induction in HepG2 cells. ${ }^{12}$ Palmitate is a weaker inducer of HSPA5, ERN1, and CHOP mRNA in human adipocytes, but the induction of GADD34, which is known to promote eIF $2 \alpha$ dephosphorylation in other tissues, was comparable to thapsigargin. Palmitate treatment triggered a 2- to 3-fold induction in the ER stress marker HSPA5 in ADHAS cell-derived adipocytes. This response is comparable to the increase of HSPA5 in human subcutaneous adipose tissues triggered by increased BMI, which is also accompanied by lower adiponectin levels. ${ }^{24}$

Previous studies have also shown that increased levels of interleukin-6 (IL-6) and TNF- $\alpha$ correlate with obesity and insulin resistance, ${ }^{29}$ suggesting a crucial role by inflammatory cytokines in the induction of ER stress, which can lead to decreased insulin sensitivity. In the present study, ER stress induction in ADHAS cell-derived adipocytes resulted in an increase in TNF- $\alpha$ mRNA expression as well as a decrease in I $\kappa \beta-\alpha$, demonstrating an increase in inflammation during ER stress. The I $\kappa$ B family of protein is known to regulate the activation of nuclear factor- $\kappa \mathrm{B}$ (NF$\kappa \mathrm{B}$ by sequestering NF- $\mathrm{KB}$ in the cytoplasm; the degradation of I $\mathrm{BB}$ proteins results in the nuclear entry of NF- $\mathrm{KB}$ dimers. The I $\mathrm{B}$ kinases (IKK) control the degradation of IкB proteins by phosphorylation, which targets the protein for proteasomal degradation. An increase in IKK activity during ER stress has been suggested as the mechanism by which ER stress may trigger inflammation (for review, see ref. 30). Thus, Iк $\beta-\alpha$ is the inhibitor of NF- $\kappa B$ activation and the decrease in the levels of Iк $\beta-\alpha$ following the induction of ER stress observed by us in this study is indicative of NF- $\mathrm{KB}$ activation. In endothelial cells, increased levels of
TNF- $\alpha$ activated NF- $\kappa B$, which is an indicator of inflammation and the addition of adiponectin inhibited TNF-

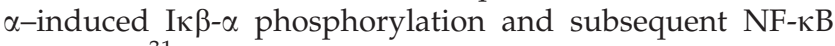
activation. ${ }^{31}$ In adipose tissues, TNF- $\alpha$ inhibited lipogenesis and adiponectin expression via inhibition of peroxisome proliferator-activated receptor- $\gamma$ (PPAR- $\gamma$ )-mediated mechanisms. ${ }^{32}$

It has also been suggested that NF-KB activation modulates antiapoptotic genes, and the fate of the cell exposed to several cytokines is determined by the integration of signals among different effectors. ${ }^{33}$ It is likely that ER stress triggers TNF- $\alpha$ cytokine secretion, which in turn triggers the inflammatory process as well as dedifferentiation of the adipocyte related to PPAR- $\gamma$-mediated effects. It is not completely clear how ER stress triggers the inflammatory process and the decrease in adiponectin; however, it is known that adiponectin has antiinflammatory properties in several cell types. ${ }^{34}$

Because we observed key differences in the induction of ER stress genes by tunicamycin and thapsigargin as compared to palmitate, we speculate that palmitate may be triggering a different signaling mechanism as compared to that of tunicamycin and/or thapsigargin. In addition to the activation of different ER stress pathways, tunicamycin or thapsigargin treatments decreased adiponectin mRNA and increased chaperone proteins ERP44 and ERO1, but palmitate treatment of human adipocytes did not alter adiponectin mRNA levels or cause significant changes in levels of ERP44. However, all three agents-tunicamycin, thapsigargin, and palmitate-decreased adiponectin protein in adipocytes as well as altered adiponectin multimerization. Induction of ER stress in adipocytes also triggered an inflammatory response, as indicated by the increase in TNF- $\alpha$ mRNA and decrease in Iк $\beta-\alpha$ protein in adipocytes. Our studies indicate that although all these agents induced ER stress, the degree of ER stress and mechanisms involved may not be the same.

Taken together, the results of the present study suggest that ER stress causes upregulation of the expression of ER stress-associated genes in ADHAS cell-derived adipocytes. The response appears to be inducer-specific in that tunicamycin and thapsigargin are stronger inducers of ER stress markers, whereas palmitate, a more physiological inducer, shows a milder effect, perhaps simulating obesity-associated states. Whether ER stress-induced downregulation of adiponectin might directly contribute to the development of an inflammatory state will be of interest in future studies.

\section{Acknowledgments}

This study was supported by Merit Review Grants (G.R., S.C.E.) from the Veterans Administration, Wake Forest School of Medicine development funds (S.C.E., S.K.D.), and National Institutes of Health (NIH) grants UL1 RR029884, R01DK71346, and R01DK71349 (R.E.M.). This manuscript is dedicated to the memory of late Dr. Steven C. Elbein.

\section{Author Disclosure Statement}

No competing financial interests exist.

The results and conclusions presented here are solely the opinion of the authors and do not necessarily reflect the opinion of the Food and Drug Administration. 


\section{References}

1. Ozcan U, Cao Q, Yilmaz E, et al. Endoplasmic reticulum stress links obesity, insulin action, and type 2 diabetes. Science 2004;306:457-461.

2. Wu J, Kaufman RJ. From acute ER stress to physiological roles of the unfolded protein response. Cell Death Differ 2006;13:374-384.

3. Oh DK, Ciaraldi T, Henry RR. Adiponectin in health and disease. Diabetes Obes Metab 2007;9:282-289.

4. Berg AH, Scherer PE. Adipose tissue, inflammation, and cardiovascular disease. Circ Res 2005;96:939-949.

5. Wang Y, Lam KS, Yau MH, Xu A. Post-translational modifications of adiponectin: Mechanisms and functional implications. Biochem J 2008;409:623-633.

6. Pajvani UB, Hawkins M, Combs TP, et al. Complex distribution, not absolute amount of adiponectin, correlates with thiazolidinedione-mediated improvement in insulin sensitivity. J Biol Chem 2004;279:12152-12162.

7. Tonelli J, Li W, Kishore P, et al. Mechanisms of early insulinsensitizing effects of thiazolidinediones in type 2 diabetes. Diabetes 2004;53:1621-1629.

8. Nakatani Y, Kaneto H, Kawamori D, et al. Involvement of endoplasmic reticulum stress in insulin resistance and diabetes. J Biol Chem 2005;280:847-851.

9. Rodbell M. Metabolism of isolated fat cells. I. Effects of hormone on glucose metabolism and lipolysis. J Biol Chem 1964;239:375-380.

10. Di Gregorio GB, Yao-Borengasser A, Rasouli N, et al. Expression of CD68 and macrophage chemoattractant protein1 genes in human adipose and muscle tissues: Association with cytokine expression, insulin resistance, and reduction by pioglitazone. Diabetes 2005; 54:2305-2313.

11. Van Harmelen.V., Skurk T, Rohrig K, et al. Effect of BMI and age on adipose tissue cellularity and differentiation capacity in women. Int J Obes Relat Metab Disord 2003; 27:889-895.

12. Das SK, Chu WS, Mondal AK, et al. Effect of pioglitazone treatment on endoplasmic reticulum stress response in human adipose and in palmitate-induced stress in human liver and adipose cell lines. Am J Physiol Endocrinol Metab 2008; 295:E393-E400.

13. Wang ZV, Schraw TD, Kim JY, et al. Secretion of the adipocyte-specific secretory protein adiponectin critically depends on thiol-mediated protein retention. Mol Cell Biol 2007;27:3716-3731.

14. Boden G, Duan X, Homko C, et al. Increase in endoplasmic reticulum (ER) stress related proteins and genes in adipose tissue of obese, insulin resistant individuals. Diabetes 2008; 57:2438-2444.

15. Yamauchi T, Kamon J, Waki H, et al. The fat-derived hormone adiponectin reverses insulin resistance associated with both lipoatrophy and obesity. Nature Med 2001;7:941-946.

16. Altomonte J, Harbaran S, Richter A, et al. Fat depot-specific expression of adiponectin is impaired in Zucker fatty rats. Metab Clin Exper 2003;52:958-963.

17. Hosogai N, Fukuhara A, Oshima K, et al. Adipose tissue hypoxia in obesity and its impact on adipocytokine dysregulation. Diabetes 2007;56:901-911.

18. Zhou L, Liu M, Zhang J, et al. DsbA-L alleviates endoplasmic reticulum stress-induced adiponectin downregulation. Diabetes 2010;59:2809-2816.

19. Hotamisligil GS. Role of endoplasmic reticulum stress and c-Jun NH2-terminal kinase pathways in inflammation and origin of obesity and diabetes. Diabetes 2005;54(Suppl 2):S73S78.

20. Lowell BB, Shulman GI. Mitochondrial dysfunction and type 2 diabetes. Science 2005;307:384-387.

21. Koh EH, Park JY, Park HS, et al. Essential role of mitochondrial function in adiponectin synthesis in adipocytes. Diabetes 2007;56:2973-2981.

22. Trayhurn P, Wang B, Wood IS. Hypoxia in adipose tissue: A basis for the dysregulation of tissue function in obesity? $\mathrm{Br}$ J Nutr 2008;100:227-235.

23. Das SK, Sharma NK, Hasstedt SJ, et al. An integrative genomics approach identifies activation of thioredoxin/ thioredoxin reductase-1-mediated oxidative stress defense pathway and inhibition of angiogenesis in obese nondiabetic human subjects. J Clin Endocrinol Metab 2011;96:E1308E1313.

24. Sharma NK, Das SK, Mondal AK, et al. Endoplasmic reticulum stress markers are associated with obesity in nondiabetic subjects. J Clin Endocrinol Metab 2008;93:4532-4541.

25. Ron D, Walter P. Signal integration in the endoplasmic reticulum unfolded protein response. Nat Rev Mol Cell Biol 2007;8:519-529.

26. Kern PA, Di Gregorio GB, Lu T, et al. Adiponectin expression from human adipose tissue: Relation to obesity, insulin resistance, and tumor necrosis factor-alpha expression. Diabetes 2003;52:1779-1785.

27. Lara-Castro C, Luo N, Wallace P, et al. Adiponectin multimeric complexes and the metabolic syndrome trait cluster. Diabetes 2006;55:249-259.

28. Novoa I, Zeng H, Harding HP, et al. Feedback inhibition of the unfolded protein response by GADD34-mediated dephosphorylation of eIF2alpha. J Cell Biol 2001;153:1011-1022.

29. Bastard JP, Maachi M, Lagathu C, et al. Recent advances in the relationship between obesity, inflammation, and insulin resistance. Eur Cytokine Netw 2006;17:4-12.

30. Bakkar N, Guttridge DC. NF-kappaB signaling: A tale of two pathways in skeletal myogenesis. Physiol Rev 2010;90: 495-511.

31. Ouchi N, Kihara S, Arita Y, et al. Adiponectin, an adipocytederived plasma protein, inhibits endothelial NF-kappaB signaling through a cAMP-dependent pathway. Circulation 2000;102:1296-1301.

32. Ruan H, Pownall HJ, Lodish HF. Troglitazone antagonizes tumor necrosis factor-alpha-induced reprogramming of adipocyte gene expression by inhibiting the transcriptional regulatory functions of NF-kappaB. J Biol Chem 2003;278: 28181-28192.

33. Kassardjian A, Kreydiyyeh SI. JNK modulates the effect of caspases and NF-kappaB in the TNFalpha-induced downregulation of $\mathrm{Na}^{+} / \mathrm{K}^{+}$ATPase in HepG2 cells. J Cell Physiol 2008;216:615-620.

34. Neumeier M, Weigert J, Schaffler A, et al. Different effects of adiponectin isoforms in human monocytic cells. J Leukoc Biol 2006;79:803-808.

Address correspondence to: Dr. Gouri Ranganathan, Ph.D. Department of Geriatrics University of Arkansas for Medical Sciences 4301 West 7th Street Little Rock, AR 72227

E-mail: ranganathangouri@uams.edu 